\title{
Dynamic Models of Some Physiological Parameters in Response to Exercise
}

\author{
Milan Stork \\ Department of Applied Electronics \\ and Telecommunications/RICE \\ University of West Bohemia, CZ \\ stork@kae.zcu.czz
}

\author{
Jaroslav Novak \\ Department of Sports Medicine \\ Medical Faculty in Plzen \\ Charles University in Prague, CZ \\ novakj@1fp.cuni.cz
}

\author{
Vaclav Zeman \\ Department of Sports Medicine \\ Medical Faculty in Plzen \\ Charles University in Prague, CZ \\ vaclav.zeman@1fp.cuni.cz
}

\begin{abstract}
The study of physiological parameters dynamic is currently the main area of research in exercise physiology. Finding dynamical models of heart rate, oxygen uptake, pulmonary ventilation and other parameters is fundamental for training methodology in sport, as well as for our knowledge of cardiorespiratory health. The present work demonstrates the application of dynamic systems models to the simulation of heart rate kinetics and oxygen consumption during workloads of time-varying intensity. The use of modern mathematical methods of analysis such as the model parameters estimation could be beneficial for understanding the relationship between the physiological responses to load and/or athletic performance, for identifying certain features in the physiological time series. As a result, the application of such methods for analysis and modeling will have a large impact not only on the development and better understanding training methodology and the testing data of athletes but also in the area of exercise medicine.
\end{abstract}

Keywords - bicycle ergometer; treadmill; heart rate; oxygen uptake; nonlinear system; optimization; state space

\section{INTRODUCTION}

For study of physiological parameters kinetics during exercise usually the treadmill or bicycle ergometers are used. The heart rate $(h r)$, oxygen uptake (VO2), $\mathrm{CO} 2$ expenditure (VCO2), pulmonary ventilation (VE) and other parameters are continuously measured within test. The results are applied in training methodology and in many cases also for medical conclusions. Using physiological parameters obtained during any form of physical activity the dynamic models can be derived. Models are described by means set of nonlinear differential equations. Most relevant is dynamics of $h r$ as a response to running velocity (on treadmill) or workload intensity (on cycle ergometer). It must be pointed out that besides of workload intensity there are other factors influencing $h r$, e.g.: ambient temperature and humidity, previous training and fatigue, over-training, altitude, medication, prodromal phase of infectious diseases, pre-start psychical state, mental activity and others.

Milan Stork's participation was supported by Department of Applied Electronics and Telecommunications, University of West Bohemia, Plzen and by the Ministry of Education, Youth and Sports of the Czech Republic under the RICE and New Technologies and Concepts for Smart Industrial Systems, project No. LO1607 and by the Internal Grant Agency of University of West Bohemia in Pilsen, Czech Republic the project SGS-2015-002 and GA15-22712S.
$\dot{V} \mathrm{O}_{2}$ is defined as the volume of oxygen used per time unit to cover energy demands of the body, either resting or during physical activity. $\dot{V} O_{2 \max }$ is the maximal capacity for oxygen consumption by the body during maximal physical exertion. Further increase in intensity doesn't yield a larger $\dot{\mathrm{V}} \mathrm{O}_{2}$. It is also known as aerobic power, maximal oxygen intake, maximal oxygen uptake, maximal oxygen consumption, aerobic capacity, and/or cardiorespiratory endurance capacity [1]. Anaerobic (lactate) threshold (LT) is defined as the point (borderline intensity of physical activity) at which the metabolic demands of physical exercise can no longer be met by valuable aerobic sources and at which an increase of anaerobic metabolism occurs, reflected by an increase of blood lactate concentration. At the intensities lower then anaerobic threshold the rate of increase in $\dot{V} O_{2}$ uptake is approximately linear function of exercise intensity, whilst at the intensities higher then the LT the function is nonlinear. $\dot{V} O_{2}$ can be measured as a time series using equipment for expired air analysis $\left(\mathrm{O}_{2}-\mathrm{CO}_{2}\right.$ analyzer $)$. The measuring of that kind is possible in the stress-test laboratory where the athlete performs either a running load on a treadmill at various speeds and/or cycling load on bicycle ergometer at various loads (in Watts) [2]. In this paper, pulmonary ventilation, breathing frequency, oxygen uptake and $\mathrm{CO}_{2}$ expenditure are measured and registered every $30 \mathrm{~s}$ (sampling period $T_{s}$ ) throughout the whole test duration.

In recent years, a few scientists have tried to develop mathematical subject in dependent models to simulate $h r$ or oxygen uptake. In [3] was presented a model formulated as a system of two nonlinear differential equations with treadmill speed as input, in [4] was presented a time-variant model specifically developed for cycling with power as input and in [5], was presented a model developed for treadmill running aiming to predict cardiovascular fitness.

The heart rate as a variable lies always within the physiological limits,

$$
h r_{\min } \leq h r(u, t) \leq h r_{\max } \quad\left[\text { beats.min } \min ^{-1}\right]
$$

There are three possible equilibria $h r(u, t)$ for the heart rate of an individual: the resting heart rate $h r(u, t)=h r_{\min }$, the maximum heart rate $h r(u, t)=h r_{\max }$, and the heart rate demand $h r(u, t)=D(u, t)$, which is related to exercise intensity $u$ (speed for treadmill, power for cycle ergometer) [6-10]. This equilibrium 
can be used to construct a model of heart rate dynamics using the following differential equation consisting of a product of three parts

$$
\begin{aligned}
& \frac{d}{d t} h r(u, t)=A_{1}\left[h r(u, t)-h r_{\min }\right]^{B_{1}} \\
& {\left[h r_{\max }-h r(u, t)\right]^{C_{1}}[D(u, t)-h r(u, t)]} \\
& \frac{d}{d t} u=I(t)
\end{aligned}
$$

where $I(t)$ is the rate of change of intensity (running velocity in $\mathrm{km} / \mathrm{h}$ and/or cycling workload in Watts), for linear changes of exercise intensities, $I(t)=c$ where $c$ is a constant and $c=0$ for the case of constant speed. The parameters $A_{l}, B_{l}, C_{l}$ in equation (2) control the shape of the curve. Parameter $A_{l}$ modifies the magnitude of the rate of change of $h r(u, t)$ and as a result the time taken to reach the steady state value. Parameter $B_{l}$ controls how quickly we leave or approach the minimum value $h r_{\text {min }}$. Parameter $C_{l}$ controls how quickly we approach or leave the maximum value $h r_{\max }$.

The experimental data, measured with sampling period $T_{\mathrm{s}}=30$ [s] were collected from a short examination of healthy male runner M1 (age 41, height $1.87 \mathrm{~m}$ and weight $88 \mathrm{~kg}$ ), who was tested on LODE treadmill, where parameters of model (obtained by optimization) were $A_{I}=6.48 .10^{-6}, B_{I}=1.63$, $C_{l}=1.57$. The result is shown in Fig. 1 .

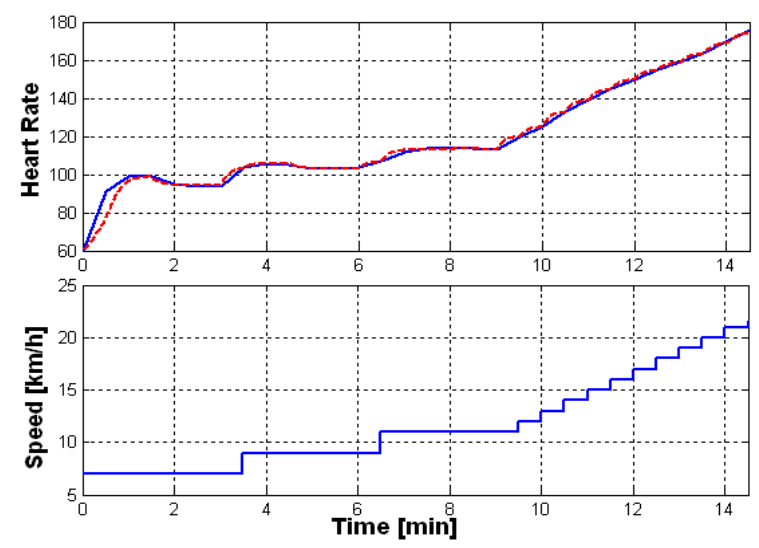

Figure 1. Measured $h r$ (solid, blue) and estimated $h r$ (dash, red, top), treadmill speed (bottom), subject M1. Solution based on nonlinear equation (2)

The estimation result presented in Fig. 1 is good, but approach based on state space models estimation which is described in this paper, because it can brings some advantages.

\section{MATERIALS AND METHODS}

A state-space representation is a mathematical model of a physical system as a set of input, output and state variables related by first-order differential equations. The state of the system can be represented in vector forms with input $\boldsymbol{u}$, state $\boldsymbol{x}$ and output $\boldsymbol{y}$

$$
\begin{aligned}
& \dot{\boldsymbol{x}}=A \boldsymbol{x}+B \boldsymbol{u} \\
& \boldsymbol{y}=C \boldsymbol{x}+D \boldsymbol{u}
\end{aligned}
$$

where matrices $A, B, C, D \quad(D=0$ in this medical application) are

$$
\begin{aligned}
A & =\left[\begin{array}{cccc}
a_{11} & a_{12} & \cdots & a_{1 n} \\
a_{21} & a_{22} & \cdots & a_{2 n} \\
\vdots & \vdots & \ddots & \vdots \\
a_{n 1} & a_{n 2} & \cdots & a_{n n}
\end{array}\right] ; B=\left[\begin{array}{c}
b_{1} \\
b_{2} \\
\vdots \\
b_{n}
\end{array}\right] \\
C & =\left[\begin{array}{llll}
c_{1} & c_{2} & \cdots & c_{n}
\end{array}\right] ; D=0
\end{aligned}
$$

Before model estimation, the data were resampled at a higher rate using lowpass interpolation. The first model, therefore parameters (matrices $A, B, C$ ) and initial conditions were estimated. After, the first model was converted to real block diagonal form. In real diagonal form, the complex eigenvalues are in 2-by-2 blocks on the diagonal. The example for $4^{\text {th }}$ order system (matrix $A$ ), with 2 real poles and 2 complex conjugate poles is described in eq. (5).

$$
A=\left[\begin{array}{cccc}
a_{11} & 0 & 0 & 0 \\
0 & a_{22} & 0 & 0 \\
0 & 0 & a_{33} & a_{34} \\
0 & 0 & -a_{34} & a_{44}
\end{array}\right]
$$

Real block diagonal form is simple for solving differential equation in time domain. Result, $h r$ estimation versus speed (for the same example subject M1, resampled data) is shown in Fig. 2.

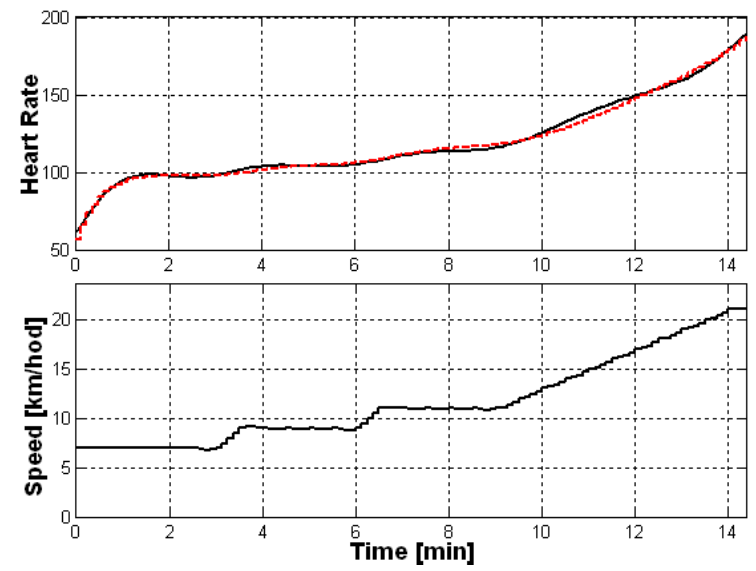

Figure 2. Measured $h r$ (solid, black) and estimated $h r$ (dash, red, top), treadmill speed (bottom), subject M1. Solution based on state space approach

State space continuous time model is (for all state space models, instead $T_{S}=0.5 \mathrm{~min}, T_{S}=0.5 \mathrm{sec}$ is used)

$$
\begin{aligned}
& \frac{d x_{1}}{d t}=-0.122 x_{1}+0.0066 u ; x_{1}(0)=0.53 \\
& \frac{d x_{2}}{d t}=-1.846 x_{1}+0.642 u ; \quad x_{2}(0)=1.66 \\
& y=144 \cdot x_{1}-11.5 \cdot x_{2}
\end{aligned}
$$
is

Time solution of $x_{1}(t)$ and $x_{2}(t)$ for unit step $(u=1)$

$$
\begin{aligned}
& x_{1}(t)=0.42 \cdot \exp (-0.122 \cdot t)+0.054 \\
& x_{2}(t)=2.0 \cdot \exp (-1.846 \cdot t)-0.348
\end{aligned}
$$


from this result can be seen that time solution consists from slow and fast part (top and middle), shown in Fig. 3.

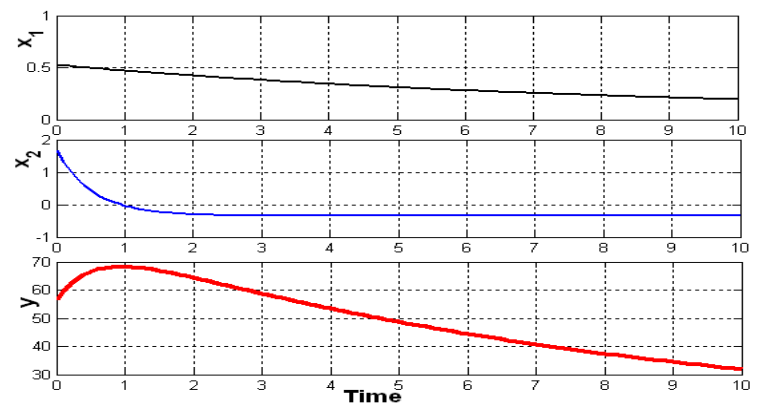

Figure 3. Time evolution of eq.(7), $x_{1}$ - top, $x_{2}$-middle, $y$ - bottom, subject M1. Solution based on state space approach

The second example is for the same subject M1, but for prolonged endurance running performance (approx. 2 hour). For this example, for $h r$ model the $5^{\text {th }}$ order of state space model must be used. Matrix A is

$$
A=\left[\begin{array}{ccccc}
-5.33 & 0 & 0 & 0 & 0 \\
0 & -1.5 & 3.87 & 0 & 0 \\
0 & -3.87 & -1.5 & 0 & 0 \\
0 & 0 & 0 & -0.003 & 0 \\
0 & 0 & 0 & 0 & -1.24
\end{array}\right]
$$

therefore solution consists from 3 real poles and 2 complex conjugate poles

$$
\begin{aligned}
p= & {[-5.33 ;-1.4980+3.8712 i ;} \\
& -1.4980-3.8712 i ;-1.24 ;-0.003]
\end{aligned}
$$

Time solution of $x_{2}(t)$ and $x_{3}(t)$ (complex conjugate poles) for unit step $(u=1)$ are

$$
\begin{aligned}
& x_{2}(t)=0.089 \cdot \cos (3.87 t) /[\exp (t)]^{3 / 2} \\
& -0.08 \cdot \sin (3.87 t) /[\exp (t)]^{3 / 2}+0.01 \\
& x_{3}(t)=-0.08 \cdot \cos (3.87 t) /[\exp (t)]^{3 / 2} \\
& -0.089 \cdot \sin (3.87 t) /[\exp (t)]^{3 / 2}-0.01
\end{aligned}
$$

The simulation results are shown in Fig. 4 and 5 . In Fig. 5 the Bode diagram of linearized system is presented.
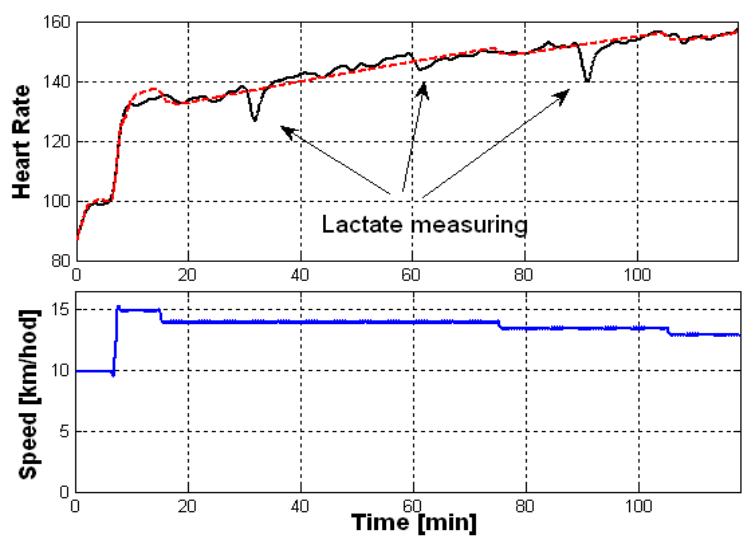

Figure 4. Measured $h r$ (solid, black) and estimated $h r$ (dash, red, top), treadmill speed (bottom), subject M1. Solution based on state space approach

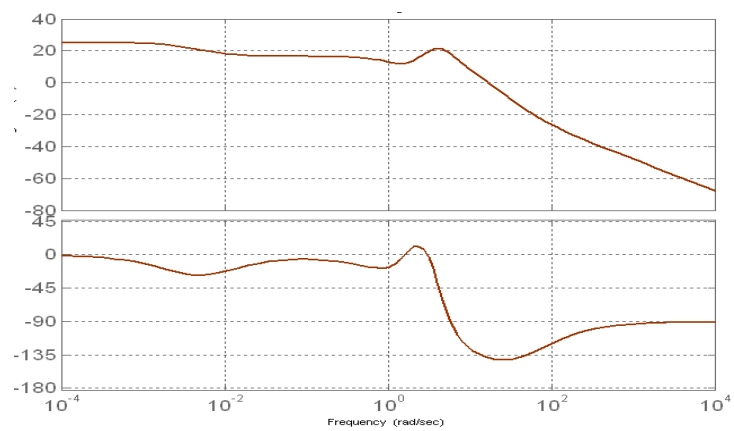

Figure 5. Bode diagram, magnitude (top) and phase (bottom) of subject M1 during during prolonged endurance exercise (running)

Next example is based on the stress test with stepvice increased workload up to the exhaustion in healthy female cross-country skier F1 (age 38, height $1.73 \mathrm{~m}$ and weight $61 \mathrm{~kg}$ ), tested on LODE bicycle ergometer. The $3^{\text {th }}$ order model is sufficient for $\mathrm{hr}$ modeling. The system is described by matrix $A$

$$
A=\left[\begin{array}{ccc}
-0.029 & 0 & 0 \\
0 & -0.43 & 0 \\
0 & 0 & -2.056
\end{array}\right]
$$

which means 3 real poles, in time domain 3 exponentials (slow, middle speed and fast) with different time constants. Result of estimation is shown in Fig. 6.
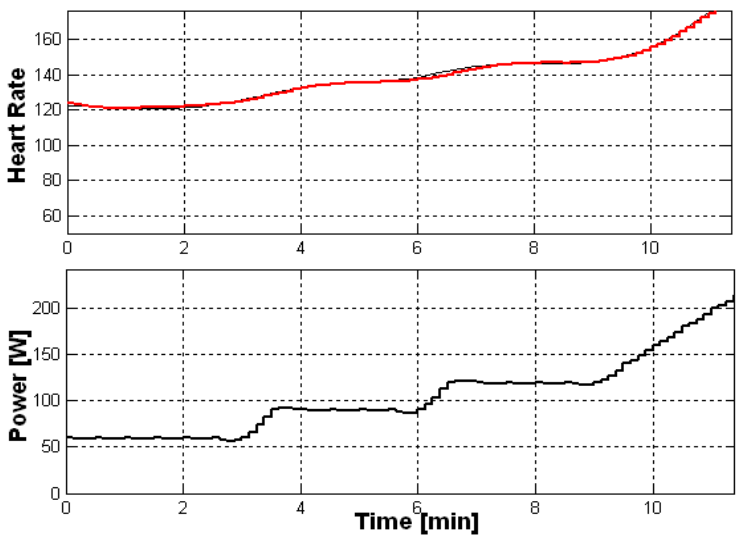

Figure 6. Measured $h r$ (solid, black) and estimated $h r$ (dash, red), top versus bicycle ergometer power (bottom), subject F1. Solution based state space approach

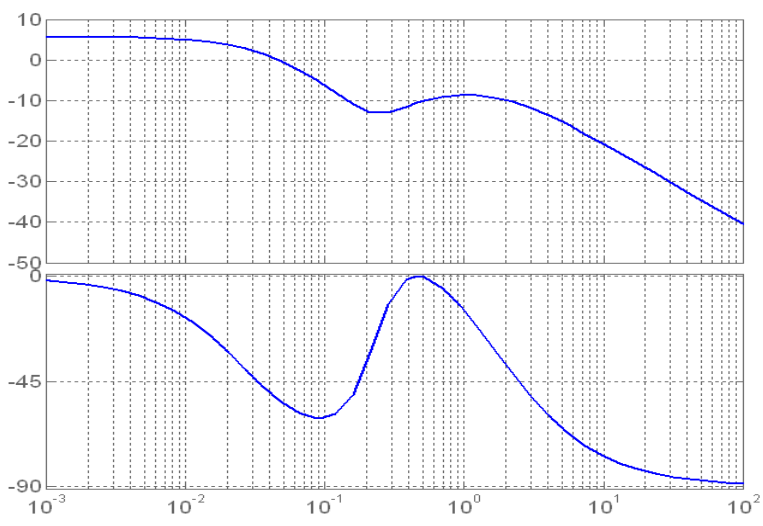

Figure 7. Bode diagram, magnitude (top) and phase (bottom) of subject F1 
It must be pointed out that also other parameters can be modeled. In next example for the same person (F1) oxygen uptake versus load is identified. Matrix $A$ is

$$
A=\left[\begin{array}{ccc}
-2.88 & 0 & 0 \\
0 & -0.34 & 0.74 \\
0 & -0.74 & -0.34
\end{array}\right]
$$

therefore system has 1 real pole and 2 complex conjugate poles. Result of estimation of oxygen consumption is displayed in Fig. 8.
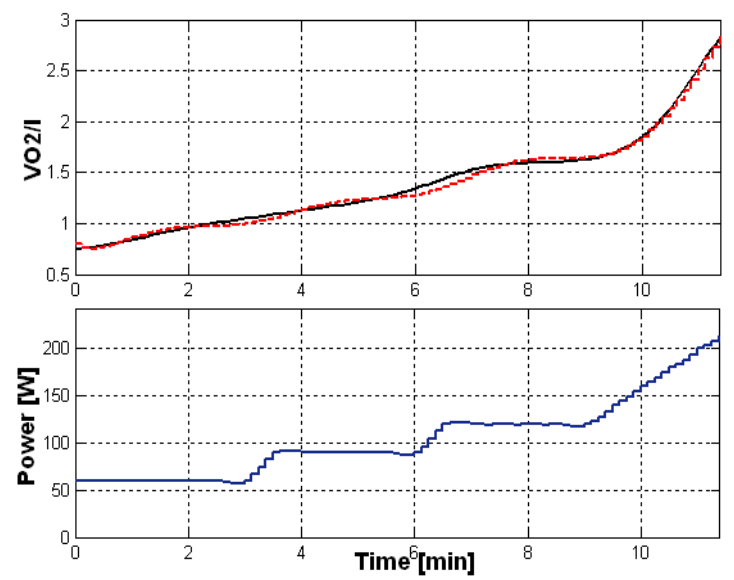

Figure 8. Measured oxygen consumption (solid, black) and estimated (dash, red), top versus bicycle ergometer power (bottom), subject F1. Solution based state space approach

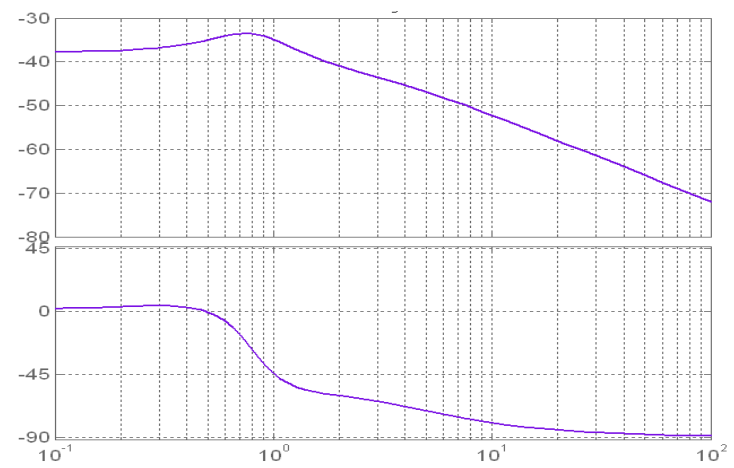

Figure 9. Bode diagram, magnitude (top) and phase (bottom) of subject F1 during exercise on bicycle ergometer

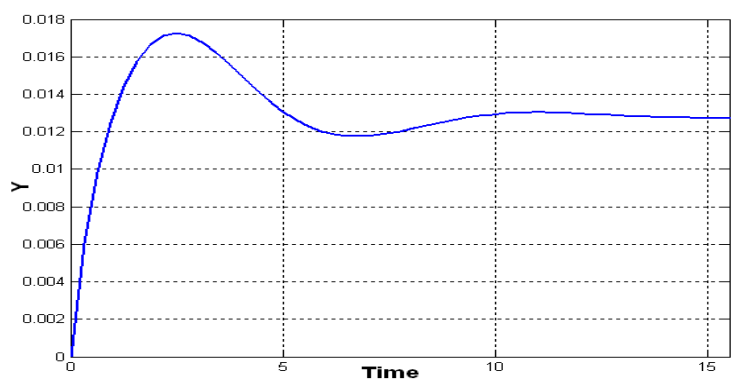

Figure 10. Step response of subject F1 during exercise on bicycle ergometer
The bode diagram and step response for model of F1 is displayed in Fig. 9 and Fig. 10 respective.

\section{RESULTS}

The aim of this paper was primarily to verify the usefulness of systems approach to modeling and analyzing the physiological response of the body to exercise. It was experimentally demonstrated that dynamic changes of selected physiological parameters obtained during different ergometric physical workloads can be simulated by set of first order differential equations with good agreement.

\section{DISCUSSION}

It is evident that there are many open problems in the field of fitness determination (intensity, duration, procedure, choice of equipment, laboratory or field etc.). The application of more modern and sophisticated techniques of analysis and modeling could provide very interesting results.

\section{REFERENCES}

[1] J. H. Wilmore and D. L. Costill," Physiology of Sport and Exercise (3rd Ed.)," Champaign, Ill.: Human Kinetics, 2004, ISBN: 0736044892

[2] K. Wasserman, J. E. Hansen, D. Y. Sue, R. Casaburi, and B. J. Whipp, "Principles of exercise testing and interpretation, including pathophysiology and clinical applications," Lippincott Williams and Wilkins, 1999.

[3] T. M Cheng, A. V Savkin, B. G Celler, S. W Su, and Lu Wang, "Nonlinear Modeling and Control of Human Heart Load Intensities," IEEE Trans. Biomed. Eng., 55(11):2499\{2508, 2008.

[4] J. Lefever, D. Berckmans, and .J M. Aerts, "Time-variant modelling of heart rate responses to exercise intensity during road cycling," Eur. J. Sport Sci., 14 Suppl 1(December):17, Jan 2012.

[5] M. S Zakynthinaki, "Modelling heart rate kinetics."PLoS One, 10(4):e0118263, Jan 2015.

[6] J. R Stirling,.M. Zakynthinaki, I. Refoyo, and J. Sampedro,. "A model of heart rate kinetics in response to exercise," Journal of Nonlinear Mathematical Physics, 15, pp. 426-436, 2008.

[7] J. R. Stirling M. Zakynthinaki, V. Billat, "Modelling and analysis of the effect of training on $\mathrm{VO}_{2}$ kinetics and anaerobic capacity,“. Bull. Math. Biol., 70(5): pp. 1348-1370, 2008. DOI:10.1007/s11538-008-9302-9.

[8] B. M. Nes, I. Janszky, U. Wisloff, A. Stoylen, and T. Karlsen, "Age-predicted maximal heart rate in healthy subjects: The HUNT Fitness Study. Scand. J. Med. Sci. Sports, 23(6):697704, dec. 2013

[9] M. J. Mazzoleni, C. L. Battaglini, K. J. Martin, E. M. Coman, and B. P. Mann,."'Modeling and predicting heart rate dynamics across a broad range of transient exercise intensities during cycling,". Sport. Eng., 2016.

[10] P. O. Astrand, K. Rodahl, H. A. Dahl, S. B. Stromme, "Text book of Work Physiology: Physiological of Bases of Exercise," Human Kinetics, 2003. 